ACTA UNIVERSITATIS LODZIENSIS

Folia Litteraria Romanica 15, 2020

https://doi.org/10.18778/1505-9065.15.22

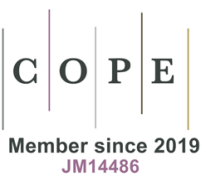

Ewa Tartakowsky

Institut des Sciences sociales du Politique (UMR 7220)

Centre de civilisation française et d'études francophones

de l'Université de Varsovie

etartakowsky@yahoo.fr

\title{
Laure Lévêque, Jules Verne. Un lanceur d'alerte dans le meilleur des mondes, Paris, L'Harmattan, coll. « Histoire, Textes, Sociétés », 2019, 217 p.
}

Dans son ouvrage dense et documenté, Laure Lévêque déconstruit le mythe d'un Jules Verne « chantre du progrès scientifique, magnifié par la figure de l'ingénieur conquérant » (p. 7). Dès ses premiers écrits, en effet, l'écrivain se révèle critique devant « les merveilleuses machines [qui] tournent aux engins de mort et l'expansion indéfinie à l'expansionnisme et à la rapacité » (Ibid.). Face à cette tension qui traverse l'œuvre vernienne, la critique littéraire, dont celle portée par Arthur B. Evans ${ }^{1}$, s'est longtemps contentée de la réduire à un partage en distinguant un premier corpus allant jusqu'à 1885, tout acquis au progrès technique, d'un second allant de 1886 (année de la mort de son éditeur Jules Hetzel) à sa mort en 1905, caractérisé par un fort techno-scepticisme. Or, et l'auteure le démontre avec finesse, c'est bien toute l'œuvre de Verne qui est traversée par cette dialectique oscillante entre la valorisation du progrès scientifique et la dénonciation de ses dangers sous-jacents. «Étonnante, une telle occultation n'est pas loin de relever de la falsification, qui promeut en Jules Verne l'apôtre du positivisme, en oubliant le lanceur d'alerte » (p. 9).

Inscrivant sa réflexion dans la filiation des travaux de Francis Lacassin, de Françoise Gaillard, de Daniel Compère, de Jean-Pierre Picot ou encore de Jean Chesneaux, ce dernier précurseur d'une lecture politique de l'œuvre vernienne ${ }^{2}$, Laure Lévêque entame son exploration par une analyse détaillée et sensible de Paris au XX ${ }^{\mathrm{e}}$ siècle. Ce roman, exhumé en 1989 et publié seulement en 1994, est l'un des premiers d'un jeune Verne (vers 1860). Ce Paris au XXe siècle, refusé par son éditeur Hetzel,

\footnotetext{
1 A. B. Evans, Jules Verne Rediscovered: Didactism and the Scientific Novel, Westport, Greenwood Press, 1988.

2 J. Chesneaux, Une lecture politique de Jules Verne, Paris, F. Maspero, 1971.
} 
n'est pas aux yeux de ce dernier conciliable avec des « récits d'aventures exaltant les conquêtes toujours plus spectaculaires de l'humanité compatibles avec l'obtention du Prix Montyon » (p. 12).

C'est que, dans cette projection du Paris des années 1960, Verne déploie déjà tous les thèmes de son scepticisme quant à l'usage du progrès technique par l'homme : « un individualisme forcené, une société férocement inégalitaire, soumise à un étroit contrôle social qu'exerce une caste de banquiers et de publicitaires que leur mainmise sur le capital et l'information met en position de monopole » (p. 18-19). L'ouvrage révèle un Jules Verne anticapitaliste, critique féroce de la spéculation immobilière dont la Compagnie Impériale Générale Immobilière qui « possédait à peu près tout Paris [et] se trouvait également propriétaire des principales villes de France » (Paris au $X X^{\mathrm{e}}$ siècle, p. 75-76 ${ }^{3}$ ) n'est qu'un avatar. Dans ce monde où l'intérêt public est absent de l'horizon politique, l'engagement politique n'est qu'une illusion soumise aux intérêts des profits financiers. L'éducation n'est pas davantage épargnée, livrée qu'elle est à une Société Générale de Crédit Instructionnel qui fait primer sur les humanités les sciences appliquées, plus utilitaires et directement employables par les gestionnaires de cette société du futur. C'est que, à travers cette dystopie, c'est bien la notion même d'un progrès inspiré par le monde de la finance qu'interroge Verne dans cet opus prémonitoire.

Au cœur de cette critique, la guerre occupe une place prépondérante, passionne des « savants fous » mais aussi, et surtout, les « impérialismes d'État». L'auteur semble même avoir transfiguré littérairement tous les conflits de son temps (p. 39). Dès 1865 , dans De la Terre à la lune, le ton est posé : «Il est évident que l'unique préoccupation de cette société savante [le Gun-Club dont le nom est en soi un programme et fait figure d'ancêtre de la National Rifle Association] fut la destruction de l'humanité, et le perfectionnement des armes de guerre, considérées comme instruments de civilisation » (De la Terre à la lune, p. 7). Comment ne pas voir dans ces propos une réflexion sur le danger d'une militarisation poussée par la volonté de puissance, via des politiques d'exploitation et des pratiques esclavagistes? Pour Verne, la seule « guerre juste » est celle de libération. Il y consacre six romans, inspirés des conflits nés du printemps des peuples de 1848 mais aussi des conflits extra-européens, telle la révolte des Cipayes de 1857 en Inde dans La maison à vapeur de 1880. Mais s'il se pose en critique de l'impérialisme britannique, il reste, comme le note Laure Lévêque, silencieux sur l'expansion coloniale française jusqu'à son dernier ouvrage d'anticipation L'Invasion de la mer, ouvrage tardif, publié en 1905.

Alors, Jules Verne serait-il promoteur avant l'heure de la notion de communs, si débattue actuellement? C'est ce qui ressort de certains propos sur la mise en commun des savoirs - « Les résultats obtenus par un savant ne lui appartiennent pas en propre ! Ils font partie du patrimoine de tous ! ( (L'Étoile du Sud, p. 177) -, ainsi que sur 3 Les citations de romans de Jules Verne renvoient, comme chez Laure Lévêque, à leur version numérique
par la Bibliothèque électronique du Québec (https://beq.ebooksgratuits.com/vents/verne.htm). 
la propriété foncière - «L Lorsque les récoltes sont engrangées, est-ce que le sol ne devrait pas appartenir à tout le monde ? [...] en me plaçant du point de vue du droit commun, il me semble que le gibier, comme l'eau des rivières, l'air de l'espace, est à tous et pour tous » (nouvelle Dix heures en chasse, 1881).

Face à un monde en phase de mondialisation féroce, où la loi du marché est devenue omniprésente qu'il s'agisse du commerce des matières premières, des savoirs ou des affaires militaires, Verne semble, selon Laure Lévêque, « hésiter entre une solution supranationale et une réponse apatride » (p. 85) sans jamais par ailleurs renoncer à mettre en scène le héros, avec ce qui le caractérise de volontarisme, souvent voué à l'échec. De fait, seule la morale anarchiste, informée « par une image idéale du contrat social qui supporte mal l'épreuve du réel » (p. 88), présente dans L'Île mystérieuse, Vingt mille lieuses sous les mers et les Naufragés du Jonathan, serait en mesure de contrer les dérives étatiques et celles du monde de la finance.

La question du sens du progrès se pose particulièrement dans Sans dessus dessous (1889). Selon les propres termes de Verne, « c'est le bouleversement, il n'y a plus de sens » (Sans dessus dessous, p. 180). Roman d'anticipation qui voit, au détriment d'une vaste partie de l'humanité, promise à la noyade, l'intervention de la North Polar Practical Association, collusion entre la science, le capital et le politique, dans la course à la vente de la calotte glaciaire arctique. Une vente anticipant le lancement d'un projectile de la Terre à la Lune par un gigantesque canon - qui n'est pas sans rappeler la course acharnée aux armements désormais au service du marché - pour dévier de son orbite la Terre et rendre ainsi exploitable cette dernière réserve des ressources énergétiques.

Pour clore sa réflexion, ouverte avec ce Paris au XX'e siècle, Laure Lévêque nous conduit vers le monde apocalyptique de la nouvelle L'éternel Adam, publiée postmortem en 1910, où « l'humanité, dont nous sommes les seuls représentants, est en voie de régression rapide et tend à se rapprocher de la brute » (L'éternel Adam, p. 393). À cet égard, l'auteure rappelle que cette mise en garde est déjà présente bien avant la fameuse césure de 1886, comme en témoigne Cinq semaines en ballon où figure ce propos de l'auteur : « À force d'inventer des machines, les hommes se feront dévorer par elles!» (Cinq semaines en ballon, p. 191).

Cette brillante monographie de l'idéologie vernienne passée au prisme du militarisme, de l'impérialisme, du colonialisme, du capitalisme ou de la mondialisation est appelée à marquer le monde académique tout comme celui des passionnés de Verne. En réagissant contre une lecture figée de l'œuvre de Verne, elle ranime l'envie de lire et de relire cet auteur dont le monde qu'il dessine semble plus que jamais d'actualité : «Un monde de périls, de conflits, d'inégalités, de violence, d'exploitation. Un monde où la question du sens est désormais posée » (p. 179). À Laure Lévêque de clore cette réflexion : «Est-il trop tard pour renouer avec la sécurité en revenant sur terre, dans les zones arctiques et ailleurs? C'est ce sur quoi nous ne saurions être trop optimiste à lire les prospectives de Verne, sauf, peut-être, à faire jouer l'esprit critique qu'il nous a laissé en partage pour au plus tôt changer de cap » (p. 190). 